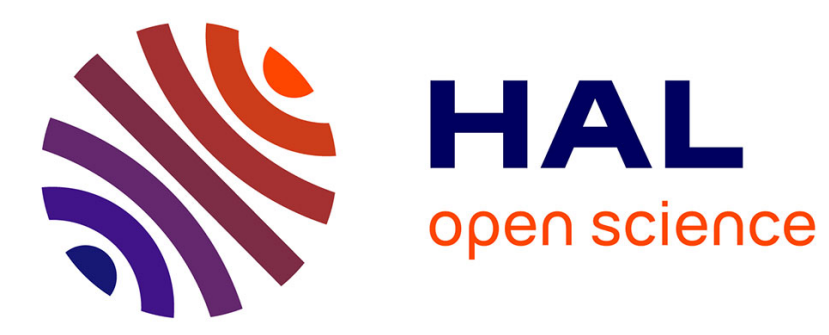

\title{
Splitting schemes for incompressible fluid/thin-walled structure interaction with unfitted meshes
}

Miguel Angel Fernández, Mikel Landajuela

\section{To cite this version:}

Miguel Angel Fernández, Mikel Landajuela. Splitting schemes for incompressible fluid/thin-walled structure interaction with unfitted meshes. Comptes Rendus. Mathématique, 2015, 353, pp.647-652. 10.1016/j.crma.2015.04.003 . hal-01095634

\section{HAL Id: hal-01095634 \\ https://inria.hal.science/hal-01095634}

Submitted on 15 Dec 2014

HAL is a multi-disciplinary open access archive for the deposit and dissemination of scientific research documents, whether they are published or not. The documents may come from teaching and research institutions in France or abroad, or from public or private research centers.
L'archive ouverte pluridisciplinaire HAL, est destinée au dépôt et à la diffusion de documents scientifiques de niveau recherche, publiés ou non, émanant des établissements d'enseignement et de recherche français ou étrangers, des laboratoires publics ou privés. 
Numerical Analysis/Mathematical Problems in Mechanics

\title{
Splitting schemes for incompressible fluid/thin-walled structure interaction with unfitted meshes ${ }^{1}$
}

\author{
Miguel A. Fernández ${ }^{\mathrm{a}, \mathrm{b}}$, Mikel Landajuela ${ }^{\mathrm{a}, \mathrm{b}}$ \\ ${ }^{\mathrm{a}}$ Inria, REO project-team, Rocquencourt - B.P. 105, F-78153 Le Chesnay cedex, France \\ ${ }^{\mathrm{b}}$ UPMC Univ Paris VI, REO project-team, UMR 7958 LJLL, F-75005 Paris, France \\ Received $* * * * * ;$ accepted after revision +++++ \\ Presented by
}

\begin{abstract}
We present two new classes of numerical methods for the solution of incompressible fluid/thin-walled structure interaction problems with unfitted meshes. The semi-implicit or explicit nature of the splitting in time is dictated by the order in which the spatial and time discretizations are performed. Stability and optimal accuracy are achieved without restrictive CFL conditions or correction iterations. To cite this article: A.Name1, A. Name2, C. R. Acad. Sci. Paris, Ser. I 340 (2005).
\end{abstract}

\section{Résumé}

Schémas de couplage et maillages non compatibles pour l'interaction d'une structure mince avec un fluide incompressible. Nous présentons deux nouvelles classes de méthodes numériques avec des maillages non compatibles pour simuler l'interaction d'une structure mince avec un fluide incompressible. Le caractère semiimplicite ou explicite du couplage en temps dépend de l'ordre dans lequel les discrétisations spatiales et temporelles sont effectuées. Stabilité et précision optimale sont obtenues sans faire recours à des conditions CFL restrictives ou à des itérations de correction. Pour citer cet article : A. Name1, A. Name2, C. R. Acad. Sci. Paris, Ser. I 340 (2005).

\section{Version française abrégée}

Considèrons le problème (1)-(2) décrivant le couplage mécanique entre un fluide visqueux incompressible (faible Reynolds) et une structure mince (petits déplacements). Ces dernières années, des progrès majeurs

Email addresses: miguel.fernandez@inria.fr (Miguel A. Fernández), mikel.landajuela_larma@inria.fr (Mikel Landajuela).

1. Work funded by the french National Research Agency (ANR) through the EXIFSI project (ANR-12-JS01-0004). 
ont été réalisés dans le développement et l'analyse de schémas de couplage explicites (à savoir, qui ne résolvent le fluide et le solide qu'une seule fois par pas de temps) pour l'approximation de (1)-(2). Tous ces travaux (see, e.g., [3,10,7,9,5,2,11]) considèrent des maillages compatibles (Figure 1, gauche). Or, il est bien connu que pour beaucoup d'applications cette hypothèse devient critique très rapidement (grandes déviations de l'interface, structures en contact, etc). Dans le cadre de maillages non compatibles (Figure 1, droite) des schémas de couplage explicite ont été proposés et analysés dans [1] pour la méthode de la frontière immergée, et dans [4] pour une méthode de type Nitsche. L'incovénient majeur est, qu'avec ces approches, la stabilité ou la précision imposent des fortes restrictions sur le pas de temps (CFL parabolique) ou des itérations de correction. Dans cette note, nous présentons deux nouvelles méthodes numériques (Algorithmes 1 and 2) qui contournent ces difficultés. Leur caractère semi-implicite ou explicite dépend de l'ordre dans lequel les discrétisations spatiales et temporelles sont effectuées. Ces schémas généralisent (pour la première fois) les idées de [7,9] au cas des maillages non compatibles. Les résultats des Propositions 2.1 et 3.2 et de la Section 4 montrent que la stabilité et la précision optimale ne sont pas soumises à des conditions CFL restrictives ou à des itérations de correction.

\section{Introduction}

We consider a fluid-structure interaction system in which the fluid is described by the Stokes equations and the structure is assumed to behave as a linear thin membrane or shell. The fluid domain is denoted by $\Omega^{\mathrm{f}} \subset \mathbb{R}^{d}(d=2,3)$. Its boundary is partitioned as $\partial \Omega^{\mathrm{f}}=\Gamma^{\mathrm{f}} \cup \Sigma$, where $\Sigma$ represents both the fluid-structure interface and the solid domain (Figure 1, left). The exterior unit-vector normal to $\partial \Omega^{\mathrm{f}}$ is denoted by $\boldsymbol{n}$. The coupled problem reads as follows: find the fluid velocity and pressure $\boldsymbol{u}: \Omega^{\mathrm{f}} \times \mathbb{R}^{+} \rightarrow \mathbb{R}^{d}$ $p: \Omega^{\mathrm{f}} \times \mathbb{R}^{+} \rightarrow \mathbb{R}$, the solid displacement and velocity $\boldsymbol{d}: \Sigma \times \mathbb{R}^{+} \rightarrow \mathbb{R}^{d}, \dot{\boldsymbol{d}}: \Sigma \times \mathbb{R}^{+} \rightarrow \mathbb{R}^{d}$ such that

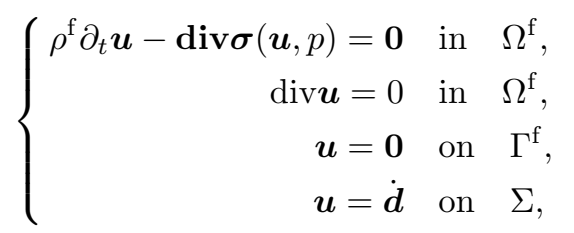$$
\left\{\begin{aligned}
\rho^{\mathrm{s}} \epsilon \partial_{t} \dot{\boldsymbol{d}}+\boldsymbol{L} \boldsymbol{d}=-\boldsymbol{\sigma}(\boldsymbol{u}, p) \boldsymbol{n} & \text { in } \quad \Sigma, \\
\dot{\boldsymbol{d}}=\partial_{t} \boldsymbol{d} & \text { in } \Sigma, \\
\boldsymbol{d}=\mathbf{0} & \text { on } \quad \partial \Sigma,
\end{aligned}\right.
$$

complemented with standard initial conditions. Here, the constants $\rho^{\mathrm{f}}$ and $\rho^{\mathrm{s}}$ stand for the fluid and solid densities, respectively, while $\epsilon$ denotes the solid thickness. The fluid Cauchy-stress tensor is given by $\boldsymbol{\sigma}(\boldsymbol{u}, p) \stackrel{\text { def }}{=}-p \boldsymbol{I}+2 \mu \boldsymbol{\varepsilon}(\boldsymbol{u})$ with $\boldsymbol{\varepsilon}(\boldsymbol{u}) \stackrel{\text { def }}{=} \frac{1}{2}\left(\boldsymbol{\nabla} \boldsymbol{u}+\boldsymbol{\nabla} \boldsymbol{u}^{\mathrm{T}}\right)$, with $\mu$ denoting the fluid dynamic viscosity. At last, the abstract surface differential operator $\boldsymbol{L}$ describes the solid elastic effects.

Over the last years, significant progress has been achieved in the development and analysis of stable and accurate explicit coupling schemes (i.e., which invoke the fluid and solid solvers only once per time-step) for the approximation of the coupled problem (1)-(2). All these studies (see, e.g., $[3,10,7,9,5,2,11])$ consider fitted meshes (Figure 1, left). For much applications, it is well known however that this assumption rapidly becomes cumbersome (e.g., large interface deflections, contacting structures, etc.). Within the unfitted mesh framework (Figure 1, right) splitting schemes of explicit nature are reported and analyzed in [1] using the finite element immersed boundary method, and in [4] for an unfitted Nitsche method. A major drawback of these approaches is that either stability or accuracy demands severe time-step restrictions (e.g., parabolic-CFL) or correction iterations. In this note, we present two new numerical methods (semi-implicit and explicit) which bypass these stability and accuracy issues. Their semi-implicit or explicit nature depends on the order in which the spatial and time discretizations are performed. These methods generalize (for the first time) the Robin-Neumann splitting paradigm of [7,9] to the unfitted mesh framework. A priori energy and error estimates are stated for some of the variants. Their performance is 
illustrated via numerical experiments in a benchmark.

\section{First discretize in space and then in time}

The first class of methods introduced and analyzed in this note combines the unfitted mesh spatial approximation of [4] with the fractional-step time-marching of $[7,9]$.

\subsection{Unfitted mesh spatial semi-discretization}

Let $\left\{\mathcal{T}_{h}^{\mathrm{s}}\right\}_{0<h<1}$ be a quasi-uniform family of triangulations of $\Sigma$. We consider a quasi-uniform family of meshes $\left\{\mathcal{T}_{h}^{\mathrm{f}}\right\}_{0<h \leq 1}$ which cover the fluid domain $\Omega^{\mathrm{f}}$. We assume that each $\mathcal{T}_{h}^{\mathrm{f}}$ is fitted to the boundary $\Gamma^{\mathrm{f}}$ but not to $\Sigma$ (Figure 1, right). The standard spaces of continuous piecewise affine func-
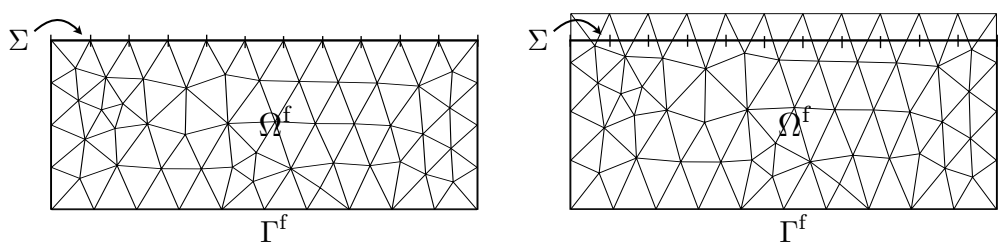

Figure 1. Fitted (left) and unfitted (right) fluid meshes.

tions associated to $\mathcal{T}_{h}^{\mathrm{s}}$ and $\mathcal{T}_{h}^{\mathrm{f}}$ will be denoted by $X_{h}^{\mathrm{s}}$ and $X_{h}^{\mathrm{f}}$, respectively. For the approximation of the fluid we will consider the spaces $\boldsymbol{V}_{h}^{\mathrm{f}} \stackrel{\text { def }}{=}\left\{\boldsymbol{v}_{h}^{\mathrm{f}} \in\left[X_{h}^{\mathrm{f}}\right]^{d} /\left.\boldsymbol{v}_{h}^{\mathrm{f}}\right|_{\Gamma^{\mathrm{f}}}=\mathbf{0}\right\}$ and $Q_{h} \stackrel{\text { def }}{=} X_{h}^{\mathrm{f}}$, while for the solid we set $\boldsymbol{V}_{h}^{\mathrm{s}} \stackrel{\text { def }}{=}\left\{\boldsymbol{v}_{h}^{\mathrm{s}} \in\left[X_{h}^{\mathrm{s}}\right]^{d} /\left.\boldsymbol{v}_{h}^{\mathrm{s}}\right|_{\partial \Sigma}=\mathbf{0}\right\}$. The following notation will also be used $a^{\mathrm{f}}\left((\boldsymbol{u}, p),\left(\boldsymbol{v}^{\mathrm{f}}, q\right)\right) \stackrel{\text { def }}{=}$ $2 \mu\left(\boldsymbol{\varepsilon}(\boldsymbol{u}), \boldsymbol{\varepsilon}\left(\boldsymbol{v}^{\mathrm{f}}\right)\right)_{\Omega_{\mathrm{f}}^{\mathrm{f}}}-\left(p, \operatorname{div} \boldsymbol{v}^{\mathrm{f}}\right)_{\Omega^{\mathrm{f}}}+(q, \operatorname{div} \boldsymbol{u})_{\Omega^{\mathrm{f}}}$ and $a^{\mathrm{s}}\left(\boldsymbol{d}, \boldsymbol{v}^{\mathrm{s}}\right) \stackrel{\text { def }}{=}\left(\boldsymbol{L} \boldsymbol{d}, \boldsymbol{v}^{\mathrm{s}}\right)_{\Sigma}$, where the symbol $(\cdot, \cdot)_{\omega}$ denotes the scalar product in $L^{2}(\omega)$. Problem (1)-(2) is approximated in space as follows: for $t>0$, find $\left(\boldsymbol{u}_{h}(t), p_{h}(t), \dot{\boldsymbol{d}}_{h}(t), \boldsymbol{d}_{h}(t)\right) \in \boldsymbol{V}_{h}^{\mathrm{f}} \times Q_{h} \times \boldsymbol{V}_{h}^{\mathrm{s}} \times \boldsymbol{V}_{h}^{\mathrm{s}}$, such that $\dot{\boldsymbol{d}}_{h}=\partial_{t} \boldsymbol{d}_{h}$ and

$$
\left\{\begin{array}{l}
\rho^{\mathrm{f}}\left(\partial_{t} \boldsymbol{u}_{h}, \boldsymbol{v}_{h}^{\mathrm{f}}\right)_{\Omega^{\mathrm{f}}}+a^{\mathrm{f}}\left(\left(\boldsymbol{u}_{h}, p_{h}\right),\left(\boldsymbol{v}_{h}^{\mathrm{f}}, q_{h}\right)\right)+\rho^{\mathrm{s}} \epsilon\left(\partial_{t} \dot{\boldsymbol{d}}_{h}, \boldsymbol{v}_{h}^{\mathrm{s}}\right)_{\Sigma}+a^{\mathrm{s}}\left(\boldsymbol{d}_{h}, \boldsymbol{v}_{h}^{\mathrm{s}}\right)+s_{h}\left(\left(\boldsymbol{u}_{h}, p_{h}\right),\left(\boldsymbol{v}_{h}^{\mathrm{f}}, q_{h}\right)\right) \\
-\left(\boldsymbol{\sigma}\left(\boldsymbol{u}_{h}, p_{h}\right) \boldsymbol{n}, \boldsymbol{v}_{h}^{\mathrm{f}}-\boldsymbol{v}_{h}^{\mathrm{s}}\right)_{\Sigma}-\left(\boldsymbol{u}_{h}-\dot{\boldsymbol{d}}_{h}, \boldsymbol{\sigma}\left(\boldsymbol{v}_{h}^{\mathrm{f}},-q_{h}\right) \boldsymbol{n}\right)_{\Sigma}+\frac{\gamma \mu}{h}\left(\boldsymbol{u}_{h}-\dot{\boldsymbol{d}}_{h}, \boldsymbol{v}_{h}^{\mathrm{f}}-\boldsymbol{v}_{h}^{\mathrm{s}}\right)_{\Sigma}=0
\end{array}\right.
$$

for all $\left(\boldsymbol{v}_{h}^{\mathrm{f}}, q_{h}, \boldsymbol{v}_{h}^{\mathrm{s}}\right) \in \boldsymbol{V}_{h}^{\mathrm{f}} \times Q_{h} \times \boldsymbol{V}_{h}^{\mathrm{s}}$. Here, $\gamma>0$ denotes the Nitsche's penalty parameter and $s_{h}$ the stabilization bilinear form $s_{h}\left(\left(\boldsymbol{u}_{h}, p_{h}\right),\left(\boldsymbol{v}_{h}^{\mathrm{f}}, q_{h}\right)\right) \stackrel{\text { def }}{=} \sum_{K \in \mathcal{T}_{h}^{\mathrm{f}}}\left[\frac{\gamma_{\mathrm{p}} h^{2}}{\mu}\left(\boldsymbol{\nabla} p_{h}, \boldsymbol{\nabla} q_{h}\right)_{K}+\gamma_{\mathrm{g}} \mu h\left(\llbracket \boldsymbol{\nabla} \boldsymbol{u}_{h} \rrbracket_{\partial K}, \llbracket \nabla \boldsymbol{v}_{h}^{\mathrm{f}} \rrbracket_{\partial K}\right)_{\partial K}\right]$, where $\llbracket \cdot \rrbracket_{\partial K}$ denotes the jump across the boundary of the element $K$ and $\gamma_{\mathrm{p}}, \gamma_{\mathrm{g}}>0$ are free parameters. As shown in [4], the unfitted space semi-discrete formulation (3) is stable and delivers optimal first-order accuracy in the energy-norm.

\subsection{Fully discrete formulation: semi-implicit coupling scheme with unfitted meshes}

In what follows, $\tau>0$ denotes the time-step length, $t_{n} \stackrel{\text { def }}{=} n \tau$ for $n \in \mathbb{N}, \partial_{\tau} x^{n} \stackrel{\text { def }}{=} \frac{1}{\tau}\left(x^{n}-x^{n-1}\right)$ stands for the first-order backward difference and $x^{\star}$ denotes the $r$-th order extrapolation, that is, $x=0$ if $r=0$, $x^{\star}=x^{n-1}$ if $r=1$ and $x^{\star}=2 x^{n-1}-x^{n-2}$ if $r=2$. The spatial semi-discrete problem (3) is discretized in time with the incremental displacement-correction scheme displayed in Algorithm 1. Note that step (4) introduces and additional unknown, the intermediate solid velocity $\dot{\boldsymbol{d}}_{h}^{n-\frac{1}{2}}$, which is implicitly coupled to 
the fluid through the solid inertial term. The remaining solid elastic contributions are treated explicitly (or ignored) in (4) via extrapolation. The end-of-step solid velocity $\dot{\boldsymbol{d}}_{h}^{n}$ is obtained by solving the solid correction step (5). The implicit treatment of the solid inertia in (4) guarantees (added-mass free) stability, while the extrapolation of the solid elastic terms introduces a certain degree of fluid-solid splitting. Note that the intermediate solid velocity $\dot{\boldsymbol{d}}_{h}^{n-\frac{1}{2}}$ cannot be eliminated in (4) and, hence, the coupling scheme is not explicit. This is a major difference with respect to the case of fitted meshes considered in $[7,9]$.

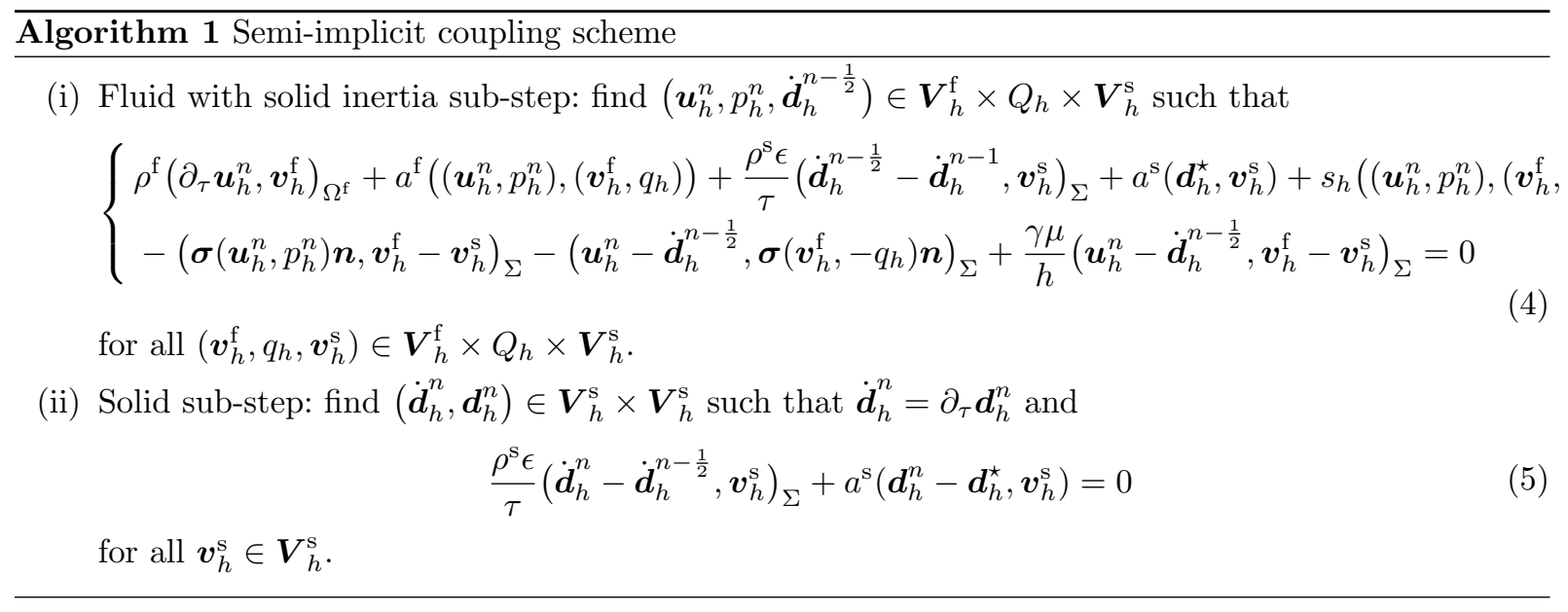

In the succeeding text, the quantities $E_{h}^{n} \stackrel{\text { def }}{=} \frac{\rho^{\mathrm{f}}}{2}\left\|\boldsymbol{u}_{h}^{n}\right\|_{0, \Omega^{\mathrm{f}}}^{2}+\frac{\rho^{\mathrm{s}} \epsilon}{2}\left\|\dot{\boldsymbol{d}}_{h}^{n}\right\|_{0, \Sigma}^{2}+\frac{1}{2} a^{\mathrm{s}}\left(\boldsymbol{d}_{h}^{n}, \boldsymbol{d}_{h}^{n}\right)$ and $\mathcal{E}_{h}^{n} \stackrel{\text { def }}{=}$ $\left(\frac{\rho^{\mathrm{f}}}{2}\left\|\boldsymbol{u}_{h}^{n}-\boldsymbol{u}\left(t_{n}\right)\right\|_{0, \Omega^{\mathrm{f}}}^{2}+\frac{\rho^{\mathrm{s}} \epsilon}{2}\left\|\dot{\boldsymbol{d}}_{h}^{n}-\dot{\boldsymbol{d}}\left(t_{n}\right)\right\|_{0, \Sigma}^{2}+\frac{1}{2} a^{\mathrm{s}}\left(\boldsymbol{d}_{h}^{n}-\boldsymbol{d}\left(t_{n}\right), \boldsymbol{d}_{h}^{n}-\boldsymbol{d}\left(t_{n}\right)\right)\right)^{\frac{1}{2}}$ respectively denote the discrete energy of the system and error at time $t_{n}$. The symbol $\lesssim$ will indicates an inequality up to a multiplicative constant (independent of $h$ and $\tau$ ). The following result, whose proof can be found in [8], states the stability and convergence properties of Algorithm 1.

Proposition 2.1 (Stability and convergence) Let $\left\{\left(\boldsymbol{u}_{h}^{n}, p_{h}^{n}, \dot{\boldsymbol{d}}_{h}^{n}, \boldsymbol{d}_{h}^{n}\right)\right\}_{n \geq 1}$ be given by Algorithm 1 . For $\gamma>0$ sufficiently large, there holds $E_{h}^{n} \lesssim E_{h}^{0}$ unconditionally for $r \in\{0,1\}$ and under the condition $\tau=\mathcal{O}\left(h^{\frac{6}{5}}\right)$ for $r=2$. Moreover, under the same conditions, there holds $\mathcal{E}_{h}^{n} \lesssim h+\tau+\tau^{2^{r-1}}$.

This result shows that Algorithm 1 preserves the stability and accuracy properties of the explicit coupling schemes introduced in $[7,9]$ with fitted meshes. Moreover, it overcomes the severe stability restrictions observed in [1] for the traditional time-marching schemes of the immersed boundary method. Algorithm 1 with $r=1$ delivers unconditional optimal overall first-order accuracy. This is also significant progress with respect to the splitting schemes reported in [4], whose accuracy is known to be non-uniform in $h$.

\section{First discretize in time and then in space}

The main drawback of Algorithm 1 is its semi-implicit nature. In other words, step (4) has a computational complexity larger than a single fluid problem, due to the additional unknown $\dot{\boldsymbol{d}}_{h}^{n-\frac{1}{2}}$. In this section, we introduce a new explicit coupling scheme which overcomes this issue without compromising stability and accuracy. The fundamental idea consists in performing the space and time discretization reversely. 


\subsection{Robin-Neumann explicit coupling schemes}

The explicit coupling schemes introduced in $[7,9]$ with fitted meshes, can be derived by applying the time splitting of Section 2.2 to the continuous problem (1)-(2). This yields:

(i) Fluid with solid inertia sub-step: find $\boldsymbol{u}^{n}: \Omega^{\mathrm{f}} \times \mathbb{R}^{+} \rightarrow \mathbb{R}^{d}, p^{n}: \Omega^{\mathrm{f}} \times \mathbb{R}^{+} \rightarrow \mathbb{R}$ and $\dot{\boldsymbol{d}}^{n-\frac{1}{2}}: \Sigma \times \mathbb{R}^{+} \rightarrow \mathbb{R}^{d}$ such that

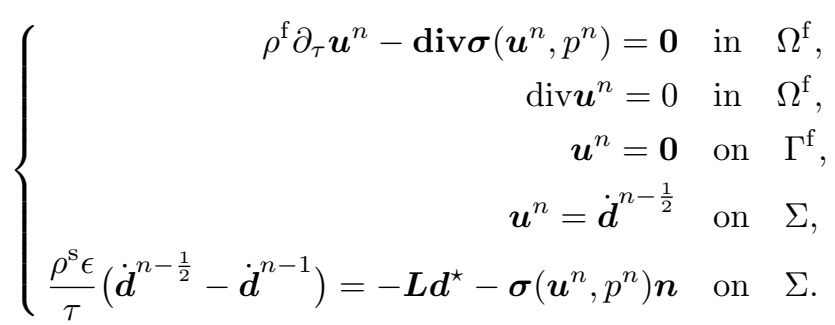

(ii) Solid sub-step: find $\boldsymbol{d}^{n}: \Sigma \times \mathbb{R}^{+} \rightarrow \mathbb{R}^{d}$ and $\dot{\boldsymbol{d}}^{n}: \Sigma \times \mathbb{R}^{+} \rightarrow \mathbb{R}^{d}$ such that $\dot{\boldsymbol{d}}^{n}=\partial_{\tau} \boldsymbol{d}^{n}$ and

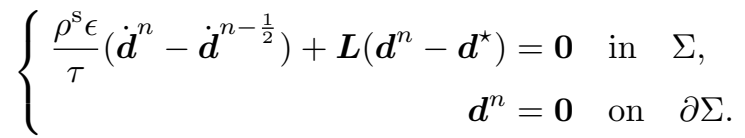

Contrarily to Algorithm 1, the intermediate solid velocity $\dot{\boldsymbol{d}}^{n-\frac{1}{2}}$ in the fluid step (i) can be eliminated through $(6)_{4}$. This yields the following explicit Robin-Neumann time splitting on the interface:

$$
\left\{\begin{aligned}
\boldsymbol{\sigma}\left(\boldsymbol{u}^{n}, p^{n}\right) \boldsymbol{n}+\kappa \boldsymbol{u}^{n}=\kappa \dot{\boldsymbol{d}}^{n-1}+\boldsymbol{g}^{\star} & \text { on } \quad \Sigma \\
\rho^{\mathrm{s}} \epsilon \partial_{\tau} \dot{\boldsymbol{d}}^{n}+\boldsymbol{L} \boldsymbol{d}^{n}=-\boldsymbol{\sigma}\left(\boldsymbol{u}^{n}, p^{n}\right) \boldsymbol{n} & \text { on } \quad \Sigma
\end{aligned}\right.
$$

with $\boldsymbol{g}^{\star} \stackrel{\text { def }}{=} \rho^{\mathrm{s}} \epsilon \partial_{\tau} \boldsymbol{d}^{\star}+\boldsymbol{\sigma}\left(\boldsymbol{u}^{\star}, p^{\star}\right) \boldsymbol{n}$ and $\kappa \stackrel{\text { def }}{=} \rho^{\mathrm{s}} \epsilon / \tau$.

\subsection{Fully discrete formulation: explicit coupling scheme with unfitted meshes}

The fundamental idea consists in performing directly an unfitted interface treatment ( $\grave{a} l a$ Nitsche) of the Robin-Neumann time splitting (8), by extending the arguments introduced in [4,6]. The proposed fully discrete scheme is based on the following result (see [8] for a proof).

Proposition 3.1 (Consistency) Let $\left\{\left(\boldsymbol{u}^{n}, p^{n}, \dot{\boldsymbol{d}}^{n}, \boldsymbol{d}^{n}\right)\right\}_{n \geq 1}$ be given by (6)-(7). Then, there holds

$$
\left\{\begin{array}{l}
\rho^{\mathrm{f}}\left(\partial_{\tau} \boldsymbol{u}^{n}, \boldsymbol{v}_{h}^{\mathrm{f}}\right)_{\Omega^{\mathrm{f}}}+a^{\mathrm{f}}\left(\left(\boldsymbol{u}^{n}, p^{n}\right),\left(\boldsymbol{v}_{h}^{\mathrm{f}}, q_{h}\right)\right)+\rho^{\mathrm{s}} \epsilon\left(\partial_{\tau} \dot{\boldsymbol{d}}^{n}, \boldsymbol{v}_{h}^{\mathrm{s}}\right)_{\Sigma}+a^{\mathrm{s}}\left(\boldsymbol{d}^{n}, \boldsymbol{v}_{h}^{\mathrm{s}}\right) \\
+\frac{\gamma \kappa \mu}{\gamma \mu+\kappa h}\left(\boldsymbol{u}^{n}-\dot{\boldsymbol{d}}^{n-1}, \boldsymbol{v}_{h}^{\mathrm{f}}-\boldsymbol{v}_{h}^{\mathrm{s}}\right)_{\Sigma}-\frac{\kappa h}{\gamma \mu+\kappa h}\left[\left(\boldsymbol{\sigma}\left(\boldsymbol{u}^{n}, p^{n}\right) \boldsymbol{n}, \boldsymbol{v}_{h}^{\mathrm{f}}-\boldsymbol{v}_{h}^{\mathrm{s}}\right)_{\Sigma}+\left(\boldsymbol{u}^{n}-\dot{\boldsymbol{d}}^{n-1}, \boldsymbol{\sigma}\left(\boldsymbol{v}_{h}^{\mathrm{f}},-q_{h}\right) \boldsymbol{n}\right)_{\Sigma}\right] \\
-\frac{h}{\gamma \mu+\kappa h}\left(\boldsymbol{\sigma}\left(\boldsymbol{u}^{n}, p^{n}\right) \boldsymbol{n}, \boldsymbol{\sigma}\left(\boldsymbol{v}_{h}^{\mathrm{f}},-q_{h}\right) \boldsymbol{n}\right)_{\Sigma}-\frac{\gamma \mu}{\gamma \mu+\kappa h}\left(\boldsymbol{g}^{\star}, \boldsymbol{v}_{h}^{\mathrm{f}}-\boldsymbol{v}_{h}^{\mathrm{s}}\right)_{\Sigma}+\frac{h}{\gamma \mu+\kappa h}\left(\boldsymbol{g}^{\star}, \boldsymbol{\sigma}\left(\boldsymbol{v}_{h}^{\mathrm{f}},-q_{h}\right) \boldsymbol{n}\right)_{\Sigma}=0
\end{array}\right.
$$

for all $\left(\boldsymbol{v}_{h}^{\mathrm{f}}, q_{h}, \boldsymbol{v}_{h}^{\mathrm{s}}\right) \in \boldsymbol{V}_{h}^{\mathrm{f}} \times Q_{h} \times \boldsymbol{V}_{h}^{\mathrm{s}}$.

A salient feature of $(9)$ is the fact that for $\kappa \rightarrow \infty$ (i.e. $\tau \rightarrow 0$ ) we formally retrieve the unfitted formulation (3). Taking successively $\boldsymbol{v}_{h}^{\mathrm{s}}=\mathbf{0}$ and $\left(\boldsymbol{v}_{h}^{\mathrm{f}}, q_{h}\right)=(\mathbf{0}, 0)$ in (9) we obtain the fully discrete method reported in Algorithm 2. Note that the resulting coupling scheme is explicit. The following result, whose proof can be found in [8], guarantees the energy stability of Algorithm 2 for $r=0$.

Proposition 3.2 (Stability) Let $\left\{\left(\boldsymbol{u}_{h}^{n}, p_{h}^{n}, \dot{\boldsymbol{d}}_{h}^{n}, \boldsymbol{d}_{h}^{n}\right)\right\}_{n \geq 1}$ be given by Algorithm 2 with $r=0$. For $\gamma>0$ sufficiently large, we have $E_{h}^{n} \leq E_{h}^{0}$. 


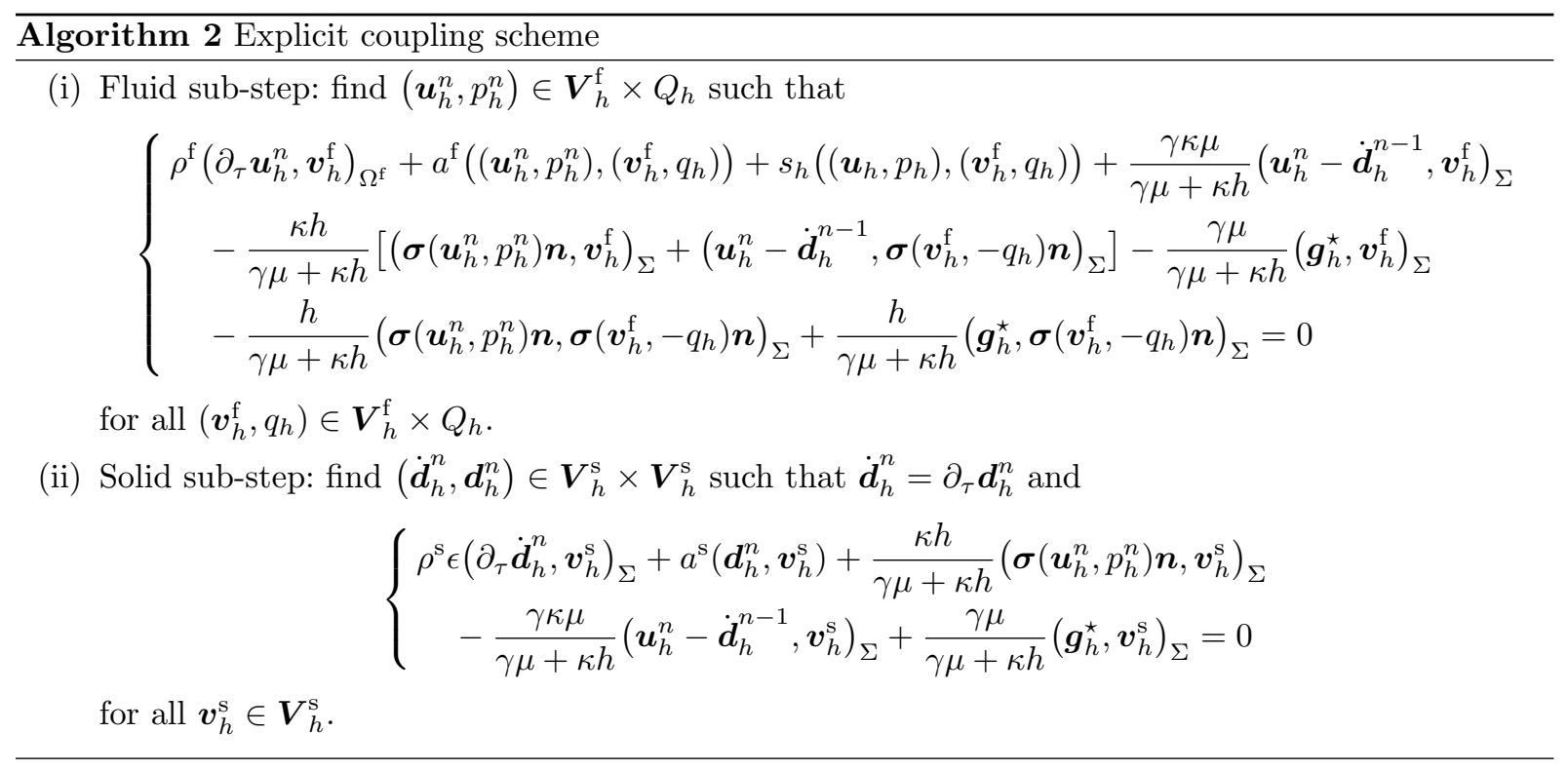

The stability of Algorithm 2 with $r=1,2$ as well as the convergence properties of all the variants are illustrated in Section 4 via numerical experiments.

\section{Numerical experiments}

In order to highlight the stability and accuracy of the proposed schemes, we consider the numerical example of [4]. We compare the results obtained with Algorithms 1 and 2 with those obtained with a first-order fully implicit scheme using fitted meshes. The convergence histories are displayed in Figure 2.
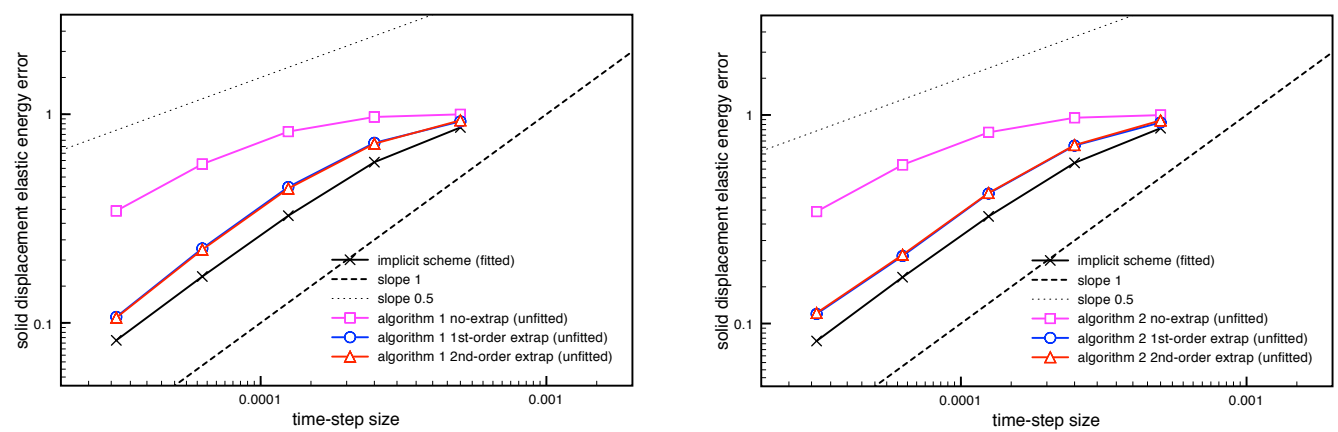

Figure 2. Time convergence history of the solid displacement in the relative elastic energy norm using Algorithm 1 (left) and Algorithm 2 (right) with $\tau=\mathcal{O}(h)$.

In order to highlight the uniformity of the convergence in $h$, we have refined both in time and in space at the same rate, $\tau=\mathcal{O}(h)$. In spite of their different semi-implicit and explicit nature, Algorithms 1 and 2 deliver practically the same behavior: stability is obtained with all the variants, optimal first-order convergence is obtained with the extrapolated variants ( $r=1,2$, unfitted) and the implicit (fitted) scheme while a sub-optimal convergence rate is exhibited by the non-extrapolated ones ( $r=0$, unfitted). 


\section{References}

[1] D. Boffi, N. Cavallini, and L. Gastaldi. Finite element approach to immersed boundary method with different fluid and solid densities. Math. Models Methods Appl. Sci., 21(12):2523-2550, 2011.

[2] M. Bukač, S. Čanić, R. Glowinski, J. Tambača, and A. Quaini. Fluid-structure interaction in blood flow capturing non-zero longitudinal structure displacement. J. Comput. Phys., 235:515-541, 2013.

[3] E. Burman and M.A. Fernández. Stabilization of explicit coupling in fluid-structure interaction involving fluid incompressibility. Comput. Methods Appl. Mech. Engrg., 198(5-8):766-784, 2009.

[4] E. Burman and M.A. Fernández. An unfitted Nitsche method for incompressible fluid-structure interaction using overlapping meshes. Comput. Methods Appl. Mech. Engrg., 279:497-514, 2014.

[5] E. Burman and M.A. Fernández. Explicit strategies for incompressible fluid-structure interaction problems: Nitsche type mortaring versus Robin-Robin coupling. Int. J. Num. Meth. Engrg., 97(10):739-758, 2014.

[6] A. Caiazzo, M.A. Fernández, J.-F. Gerbeau, and V. Martin. Projection schemes for fluid flows through a porous interface. SIAM J. Sci. Comput., 33(2):541-564, 2011.

[7] M.A. Fernández. Incremental displacement-correction schemes for incompressible fluid-structure interaction: stability and convergence analysis. Numer. Math., 123(1):21-65, 2013.

[8] M.A. Fernández and M. Landajuela. Unfitted formulations and splitting schemes for fluid/thin-walled structure interaction. In preparation.

[9] M.A. Fernández, J. Mullaert, and M. Vidrascu. Explicit Robin-Neumann schemes for the coupling of incompressible fluids with thin-walled structures. Comput. Methods Appl. Mech. Engrg., 267:566-593, 2013.

[10] G. Guidoboni, R. Glowinski, N. Cavallini, and S. Čanić. Stable loosely-coupled-type algorithm for fluid-structure interaction in blood flow. J. Comp. Phys., 228(18):6916-6937, 2009.

[11] M. Lukáčová, G. Rusnáková, and A. Hundertmark. Kinematic splitting algorithm for fluid-structure interaction in hemodynamics. Comput. Methods Appl. Mech. Engrg., 265(1):83-106, 2013. 